\title{
NOTE ON THE COMBINATORIAL FORMULA FOR ${ }_{n} H_{r}$
}

\author{
G. BAIKUNTH NATH and P. V. KRISHNA IYER
}

(Received 13th May 1969; revised 1st May 1970)

Communicated by E. S. Barnes

\section{Introduction}

${ }_{n} H_{r}$ denotes the number of $n \times n$ matrices with non-negative integral entries, with row-sums and column-sums all equal to $r$. Kenji Mano [3] investigated the number ${ }_{n} H_{r}$ in which $n$ distinct objects each replicated $r$ times can be distributed in equal numbers among $n$ cells. He gives an intricate formula for the case $r=2$. Recently, Anand et al. [1], making use of partitions, extended it to ${ }_{3} H_{r}$ and stated a plausible formula for ${ }_{n} H_{r}$.

In the present note, calculations are expedited by the use of generating functions. Explicit formulae for ${ }_{3} H_{r}$ and ${ }_{4} H_{r}$ are derived. The procedure applies to rectangular matrices as well as square ones.

\section{The formula for ${ }_{3} H_{r}$}

As stated earlier, ${ }_{3} H_{r}$ is the number of matrices

$$
\left[\begin{array}{lll}
a_{1} & a_{2} & a_{3} \\
b_{1} & b_{2} & b_{3} \\
c_{1} & c_{2} & c_{3}
\end{array}\right]
$$

with non-negative integral entries, such that row and column sums are all equal to $r$.

If the entries in the first row $\left(a_{1}, a_{2}, a_{3}\right)$ are arbitrarily fixed, then it remains to arrange the remaining $r-a_{1}, r-a_{2}$ and $r-a_{3}$ elements in the last two cells of the three columns respectively.

The generating function for such a scheme is

$$
\left(1-x^{r-a_{1}+1}\right)\left(1-x^{r-a_{2}+1}\right)\left(1-x^{r-a_{3}+1}\right)(1-x)^{-3}
$$

The coefficient of $x^{r}$ in (2.1), namely, 


$$
\left(\begin{array}{c}
r+2 \\
2
\end{array}\right)-\sum_{i=1}^{3}\left(\begin{array}{c}
a_{i}+1 \\
2
\end{array}\right)
$$

gives the number of arrangements for row and column sums all equal to $r$, conditional on the specification that row one has entries $\left(a_{1}, a_{2}, a_{3}\right)$. The value ${ }_{3} H_{r}$ then results from summing (2.2) over all non-negative integral triples $\left(a_{1}, a_{2}, a_{3}\right)$ compatible with $a_{1}+a_{2}+a_{3}=r$.

Since the first term of (2.2) is constant and does not depend on the entries $\left(a_{1}, a_{2}, a_{3}\right)$ of the specified row one, its sum over non-negative integral values is achieved by multiplying with the coefficient of $x^{r}$ in the generating function $\left(1-x^{r+1}\right)^{3}(1-x)^{-3}$, namely

$$
\left(\begin{array}{c}
r+2 \\
2
\end{array}\right)^{2}
$$

Now the sum of the second term of (2.2) is conditional with respect to the entries of the first row. Its product with the coefficient of $x^{r-a_{i}}$ in the generating function $\left(1-x^{r+1}\right)^{2}(1-x)^{-2}$, namely,

$$
\sum_{a_{i}}\left(\begin{array}{c}
a_{i}+1 \\
2
\end{array}\right)\left(r-a_{i}+1\right), \quad i=1,2,3
$$

$$
=3\left(\begin{array}{c}
r+3 \\
4
\end{array}\right)
$$

gives the required sum.

Subtraction of (2.4) from (2.3), gives

$$
\left(\begin{array}{c}
r+2 \\
2
\end{array}\right)^{2}-3\left(\begin{array}{c}
r+3 \\
4
\end{array}\right)=\left(\begin{array}{c}
r+2 \\
2
\end{array}\right)+3\left(\begin{array}{c}
r+3 \\
4
\end{array}\right)
$$

which determines the value ${ }_{3} H_{r}$.

It may be remarked, the proof given here is much shorter than that given in [1].

\section{The formula for ${ }_{4} H_{r}$}

Using the technique of Section 2, first we construct a formula for $3 \times 4$ matrices (which incidently are rectangular), then with suitable entries in the fourth row extend it to $4 \times 4$ matrices ensuring row and column sums all equal to $r$.

Consider a matrix

$$
\left[\begin{array}{llll}
a_{1} & a_{2} & a_{3} & a_{4} \\
b_{1} & b_{2} & b_{3} & b_{4} \\
c_{1} & c_{2} & c_{3} & c_{4}
\end{array}\right]
$$


with non-negative integral entries, such that the row sums are all equal to $r$ and the $i$-th column-sum is equal to $s_{i}(i=1,2,3,4), 0<s_{i} \leqq r$.

Fix the entries $\left(a_{1}, a_{2}, a_{3}, a_{4}\right)$ in the first row arbitrarily. The remaining elements $s_{i}-a_{i}(i=1,2,3,4)$ are to be arranged in the last two cells of the four columns respectively.

The coefficient of $x^{r}$ in the generating function

$$
\prod_{i}\left(1-x^{s_{i}-a_{i}+1}\right)(1-x)^{-4}
$$

is

$$
\left(\begin{array}{c}
r+3 \\
3
\end{array}\right)-\sum_{i}\left(\begin{array}{c}
r-s_{i}+a_{i}+2 \\
3
\end{array}\right)+\sum_{\substack{i, j \\
i \neq j}}\left(\begin{array}{c}
r-s_{i}-s_{j}+a_{i}+a_{j}+1 \\
3
\end{array}\right)
$$

which gives the number of arrangements for row-sums equal to $r$ and columnsums equal to $s_{i}(i=1,2,3,4)$, conditional on the specification of row one with entries $\left(a_{1}, a_{2}, a_{3}, a_{4}\right)$. The value of the $3 \times 4$ matrix then results from summing (3.2) over all non-negative integral quadruples $\left(a_{1}, a_{2}, a_{3}, a_{4}\right)$ compatible with $a_{1}+a_{2}+a_{3}+a_{4}=r$.

As in Section 2, the first term in (3.2) is constant, its sum over non-negative integral values is achieved by multiplying it with the coefficient of $x^{r}$ in the generating function

which is

$$
\prod_{i}\left(1-x^{s_{i}+1}\right)(1-x)^{-4}
$$

$$
\left(\begin{array}{c}
r+3 \\
3
\end{array}\right)\left\{\left(\begin{array}{c}
r+3 \\
3
\end{array}\right)-\sum_{i}\left(\begin{array}{c}
r-s_{i}+2 \\
3
\end{array}\right)\right\}
$$

with $s_{i}(i=1,2,3,4)$ fixed. $]$

The sum of the second term in (3.2), conditional with respect to the quadruples $\left(a_{1}, a_{2}, a_{3}, a_{4}\right)$ of the specified row one, is obtained from its product with the coefficient of $x^{r-a_{i}}$ in the generating function

$$
\prod_{k}\left(1-x^{s_{k}+1}\right)(1-x)^{-3}, k=1,2,3,4 \text { and different from } i
$$

which is, for $i=1,2,3,4$,

$$
\begin{gathered}
\sum_{a_{i}}\left(\begin{array}{c}
r-s_{i}+a_{i}+2 \\
3
\end{array}\right)\left\{\left(\begin{array}{c}
r-a_{i}+2 \\
2
\end{array}\right)-\sum_{k}\left(\begin{array}{c}
r-a_{i}-s_{k}+1 \\
2
\end{array}\right)\right\} \\
=\sum_{i}\left\{\left(\begin{array}{c}
r+3 \\
4
\end{array}\right)\left(\begin{array}{c}
r-s_{i}+1 \\
2
\end{array}\right)+\left(\begin{array}{c}
r+4 \\
5
\end{array}\right)\left(r-s_{i}\right)+\left(\begin{array}{c}
r+5 \\
6
\end{array}\right)\right. \\
\left.-\left(\begin{array}{c}
r+2 \\
2
\end{array}\right)\left(\begin{array}{c}
r-s_{i}+2 \\
4
\end{array}\right)-(r+1)\left(\begin{array}{c}
r-s_{i}+3 \\
5
\end{array}\right)+2\left(\begin{array}{c}
r-s_{i}+3 \\
6
\end{array}\right)\right\}
\end{gathered}
$$


[4]

$-\sum_{\substack{i, j \\ i \neq j}}\left\{\left(\begin{array}{c}2 r-s_{i}-s_{j}+4 \\ 6\end{array}\right)-\left(\begin{array}{c}r-s_{i}+3 \\ 5\end{array}\right)\left(r-s_{j}\right)-\left(\begin{array}{c}r-s_{i}+2 \\ 4\end{array}\right)\left(\begin{array}{c}r-s_{j}+1 \\ 2\end{array}\right)\right\}$.

Similarly, the sum of the third term in (3.2), conditional with respect to the entries of the first row, is obtained from its product with the coefficient of $x^{r-a_{i}-a_{j}}(i \neq j)$ in the generating function

$\prod_{k}\left(1-x^{s_{k}+1}\right)(1-x)^{-2}, k=1,2,3,4$; different from $i, j$ and $i \neq j$, namely

$$
\sum_{\substack{a_{i}, a_{j} \\
i \neq j}}\left(\begin{array}{c}
r-s_{i}-s_{j}+a_{i}+a_{j}+1 \\
3
\end{array}\right)\left\{\left(r-a_{i}-a_{j}+1\right)-\sum_{k}\left(r-a_{i}-a_{j}-s_{k}\right)\right\}
$$

$k$ different from $i, j ; i, j=1,2,3,4$.

For simplicity, to obtain the sum (3.5), we define

$$
u=a_{i}+a_{j}, \quad 0 \leqq u \leqq r,
$$

and the coefficient of $x^{u}$ in the generating function

is

$$
\left(1-x^{s_{i}+1}\right)\left(1-x^{s_{j}+1}\right)(1-x)^{-2}
$$

$$
(u+1)-\left(u-s_{i}\right)-\left(u-s_{j}\right), i \neq j .
$$

Taking note of the restriction on $u$, the use of (3.6) and (3.7) reduces (3.5) to

$$
\sum_{u}\left(\begin{array}{c}
r-s_{i}-s_{j}+u+1 \\
3
\end{array}\right)\left[(r-u+1)\left\{(u+1)-\left(u-s_{i}\right)-\left(u-s_{j}\right)\right\}\right], i \neq j .
$$

The other terms of (3.5) under this substitution do not exist. Performing summation over $u(3.8)$ reduces to

$$
\begin{aligned}
& \sum_{\substack{i<j \\
i, j}}\left\{-4\left(\begin{array}{c}
2 r-s_{i}-s_{j}+3 \\
6
\end{array}\right)+(r-1)\left(\begin{array}{c}
2 r-s_{i}-s_{j}+3 \\
5
\end{array}\right)\right. \\
& +4\left(\begin{array}{c}
r-s_{i}+3 \\
6
\end{array}\right)+4\left(\begin{array}{c}
r-s_{j}+3 \\
6
\end{array}\right) \\
& -\left(\begin{array}{c}
r-s_{i}+2 \\
5
\end{array}\right)\left(r-s_{j}\right)-\left(\begin{array}{c}
r-s_{j}+2 \\
5
\end{array}\right)\left(r-s_{i}\right)-\left(\begin{array}{c}
r-s_{i}+3 \\
5
\end{array}\right)\left(r-s_{j}+2\right) \\
& \left.-\left(\begin{array}{c}
r-s_{j}+3 \\
5
\end{array}\right)\left(r-s_{i}+2\right)\right\} .
\end{aligned}
$$

Subtraction of (3.4) from the addition of (3.3) and (3.9) completely determines a formula for a matrix of type $3 \times 4$. 
Now to extend the results for the matrix of order $3 \times 4$ to the one of order $4 \times 4$, we insert elements $d_{i}(i=1,2,3,4)$ in the fourth row, such that

$$
s_{i}+d_{i}=r, 0 \leqq d_{i}<r, s_{i} \neq 0 .
$$

Summation over $d_{i}$, then yields

$$
-4\left(\begin{array}{c}
r+2 \\
2
\end{array}\right)+\left(\begin{array}{c}
r+3 \\
3
\end{array}\right)-12\left(\begin{array}{c}
r+3 \\
4
\end{array}\right)+20\left(\begin{array}{c}
r+4 \\
5
\end{array}\right)+152\left(\begin{array}{c}
r+5 \\
7
\end{array}\right)+352\left(\begin{array}{c}
r+6 \\
9
\end{array}\right) \text {. }
$$

It is clear that the result (3.10) is reached without considering the possibilities of being any of the $s_{i}=0$ in the matrix of order $3 \times 4$. In such a situation, the said matrix reduces to that of order $3 \times 3$ (Section 2). The natural extension of this $3 \times 3$ matrix so obtained, to that of order $4 \times 4$ is to insert the value $r$ in one of the cells of the fourth row corresponding to the new added column, and this can surely have four possibilities. Thus, adding four times the result (2.5) to (3.10) we finally determine the value ${ }_{4} H_{r}$, i.e.,

$$
\left(\begin{array}{c}
r+3 \\
3
\end{array}\right)+20\left(\begin{array}{c}
r+4 \\
5
\end{array}\right)+152\left(\begin{array}{c}
r+5 \\
7
\end{array}\right)+352\left(\begin{array}{c}
r+6 \\
9
\end{array}\right)
$$

These results have been verified to be correct by actual computations and agree with the tables of symmetric functions [2].

\section{Acknowledgments}

We are very grateful to the referee for helpful comments and valuable suggestions.

\section{References}

[1] H. Anand, V. C. Dumir and H. Gupta, 'A Combinatorial Distribution Problem', Duke Math. J. 33 (1966), 757-769.

[2] F. N. David, and M. G. Kendall, 'Tables of Symmetric Functions: Part IV', Biometrika 40 (1953), 427-446.

[3] Kenji Mano, 'On the formula of ${ }_{n} H_{r}$ ', Scientific Reports of the Faculty of Literature and Science: Hirosaki University 8 (1961), 58-60.

Department of Mathematics

University of Queensland

Australia 